\title{
QUANTUM HOMOGENEOUS SPACES AS QUANTUM QUOTIENT SPACES
}

\author{
TOMAsz BRZEZIŃski \\ Institute of Mathematics, University of Łódź \\ ul. Banacha 22, 90-238 Eódź, Poland
}

August 1995

\begin{abstract}
We show that certain embeddable homogeneous spaces of a quantum group that do not correspond to a quantum subgroup still have the structure of quantum quotient spaces. We propose a construction of quantum fibre bundles on such spaces. The quantum plane and the general quantum two-spheres are discussed in detail.
\end{abstract}

\section{Introduction}

A homogeneous space $X$ of a Lie group $G$ may be always identified with the quotient space $G / G_{0}$, where $G_{0}$ is a Lie subgroup of $G$. When the notion of a homogeneous space is generalised to the case of quantum groups or non-commutative Hopf algebras the situation becomes much more complicated. A general quantum homogeneous space of a quantum group $H$ need not be a quotient space of $H$ by its quantum subgroup. By a quantum subgroup of $H$ we mean a Hopf algebra $H_{0}$ such that there is a Hopf algebra epimorphism $\pi: H \rightarrow H_{0}$. The quotient space is then understood as a subalgebra of $H$ of all points that are fixed under the coaction of $H_{0}$ on $H$ induced by $\pi$. A quantum homogeneous space $B$ of $H$ might be such a quotient space but it is not in general. There is, however, a certain class of quantum homogeneous spaces, of which the quantum two sphere of Podleś $[\mathrm{P} 1]$ is the most prominent example, that not being quotient spaces by a quantum subgroup of $H$, may be embedded in $H$. One terms such homogeneous spaces $\mathrm{em}$ beddable [P2]. The general quantum two sphere $S_{q}^{2}(\mu, \nu)$ is such an embeddable

Most of this paper was written during my stay at Universite Libre de Bruxelles supported by the European Union Human Capital and Mobility grant. This work is also supported by the grant KBN 2 P302 21706 p01 
homogeneous space of the quantum group $S U_{q}(2)$, and it is a quantum quotient space in the above sense when $\nu=0$. In the latter case the corresponding subgroup of $S U_{q}(2)$ may be identified with the algebra of functions on $U(1)$. In this paper we show that certain embeddable quantum homogeneous spaces, and the general quan-

tum two sphere $S_{q}^{2}(\mu, \nu)$ among them, can still be understood as quotient spaces or fixed point subalgebras. Precisely we show that there is a coalgebra $C$ and a coalgebra epimorphism $\pi: H \rightarrow C$ such that the fixed point subspace of $H$ under the coaction of $C$ on $H$ induced from the coproduct in $H$ by a pushout by $\pi$ is a subalgebra of $H$ isomorphic to $B$.

The interpretation of embeddable quantum homogeneous spaces as quantum quotient spaces allows one to develop the quantum group gauge theory of such spaces following the lines of $[\mathrm{BM}]$. The study of such a gauge theory becomes even more important once the appearance of the quantum homogeneous spaces in the A. Connes geometric description of the standard model was annonunced [C]. For this purpose, however, one needs to generalise the notion of a quantum principal bundle of $[\mathrm{BM}]$ so that a Hopf algebra playing the role of a quantum structure group there may be replaced by a coalgebra. We propose such a generalisation. Since the theory of quantum principal bundles is strictly related to the theory of Hopf-Galois extensions (cf. $[\mathrm{S}]$ ), we thus propose a generalisation of such extensions.

The paper is organised as follows. In Section 1 we describe the notation we use in the sequel. In Section 2 we show a fixed point subalgebra structure of embeddable quantum homogeneous spaces. Next we propose a suitable generalisation of the notion of a quantum principal bundle in Section 3. Sections 4 and 5 are devoted to careful study of two examples of quantum embeddable spaces, namely the quantum plane $\mathbf{C}_{q}^{2}[\mathrm{M}]$ and the quantum sphere $S_{q}^{2}(\mu, \nu)[\mathrm{P} 1]$.

\section{Preliminaries}

In the sequel all the vector spaces are over the field $k$ of characteristic not $2 . C$ denotes a coalgebra with the coproduct $\Delta: C \rightarrow C \otimes C$ and the counit $\epsilon: C \rightarrow k$ which satisfy the standard axioms, cf. $[\mathrm{Sw}]$. For the coproduct we use the Sweedler sigma notation 
where $c \in C$, and the summation sign and the indices are suppressed. A vector space $A$ is a left $C$-comodule if there exists a map $\Delta_{L}: A \rightarrow C \otimes A$, such that $(\Delta \otimes i d) \circ \Delta_{L}=\left(i d \otimes \Delta_{L}\right) \circ \Delta_{L}$, and $(\epsilon \otimes i d) \circ \Delta_{L}=i d$. For $\Delta_{L}$ we use the explicit notation

$$
\Delta_{L} a=a_{(1)} \otimes a_{(\infty)},
$$

where $a \in A$ and all $a_{(1)} \in C$ and all $a_{(\infty)} \in A$.

Similarly we say that a vector space $A$ is a right $C$-comodule if there exists a map $\Delta_{R}: A \rightarrow A \otimes C$, such that $\left(\Delta_{R} \otimes i d\right) \circ \Delta_{R}=(i d \otimes \Delta) \circ \Delta_{R}$, and $(i d \otimes \epsilon) \circ \Delta_{R}=i d$. For $\Delta_{R}$ we use the explicit notation

$$
\Delta_{R} a=a_{(0)} \otimes a_{(1)},
$$

where $a \in A$ and all $a_{(1)} \in C$ and all $a_{(0)} \in A$.

$H$ denotes a Hopf algebra with product $m: H \otimes H \rightarrow H$, unit 1, coproduct $\Delta: H \rightarrow H \otimes H$, counit $\epsilon: H \rightarrow k$ and antipode $S: H \rightarrow H$. We use Sweedler's sigma notation as before. Similarly as for a coalgebra we can define right and left $H$-comodules. For a right $H$-comodule $A$ we denote by $A^{c o H}$ a vector subspace of $A$ of all elements $a \in A$ such that $\Delta_{R} a=a \otimes 1$. We say that a right (resp. left) $H$-comodule $A$ is a right (resp. left) $H$-comodule algebra if $A$ is an algebra and $\Delta_{R}$ (resp. $\Delta_{L}$ ) is an algebra map.

A vector subspace $J$ of $H$ such that $\epsilon(J)=0$ and $\Delta J \subset J \otimes H \oplus H \otimes J$ is called a coideal in $H$. If $J$ is a coideal in $H$ then $C=H / J$ is a coalgebra with a coproduct $\Delta$ given by $\Delta=(\pi \otimes \pi) \circ \Delta$, where $\pi: H \rightarrow C$ is a canonical surjection. The counit $\epsilon$ in $C$ is defined by the commutative diagram

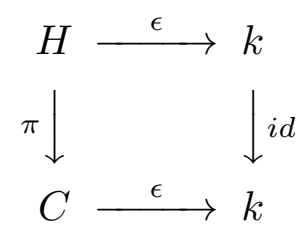

\section{Quantum homogeneous Spaces}

In this section we show that if an embeddable quantum homogeneous space satisfies certain additional assumption it may be identified with a quantum quotient 
Definition 2.1. [P2]. Let $H$ be a Hopf algebra and $B$ be a left $H$-comodule algebra with the coaction $\Delta_{L}: B \rightarrow H \otimes B$. We say that $B$ is an embeddable quantum homogeneous space or simply an embeddable $H$-space if there exists an algebra inclusion $i: B \hookrightarrow H$ such that $\Delta \circ i=(i d \otimes i) \circ \Delta_{L}$, i.e., $i$ is an intertwiner.

\section{Proposition 2.2.}

(1) A left $H$-comodule algebra $B$ is an embeddable $H$-space if and only if there exists an algebra character $\kappa: B \rightarrow k$ such that the linear map $i_{\kappa}: B \rightarrow H$, $i_{\kappa}: b \mapsto b_{(1)} \kappa\left(b_{(\infty)}\right)$ is injective.

(2) If $B$ is an embeddable $H$-space then the linear map $\chi_{L}: B \otimes B \rightarrow H \otimes B$, $\chi_{L}: b \otimes b^{\prime} \mapsto b_{(1)} \otimes b_{(\infty)} b^{\prime}$ is injective.

Proof. (1) If $B$ is an embeddable quantum homogeneous space then $\kappa=\epsilon \circ i$ is a character of $B$. Since $i$ is an intertwiner, for any $b \in B$ we compute

$$
i_{\kappa}(b)=b_{(1)} \epsilon\left(i\left(b_{(\infty)}\right)\right)=i(b)_{(1)} \epsilon\left(i(b)_{(2)}\right)=i(b),
$$

thus $i_{\kappa}$ is an inclusion.

Conversly assume that there is a character $\kappa: B \rightarrow k$ such that $i_{\kappa}$ is injective. Then clearly $i_{\kappa}$ is an algebra inclusion. Furthermore

$$
\Delta\left(i_{\kappa}(b)\right)=b_{(1)} \otimes b_{(2)} \kappa\left(b_{(\infty)}\right)=b_{(1)} \otimes i_{\kappa}\left(b_{(\infty)}\right)=\left(i d \otimes i_{\kappa}\right) \circ \Delta_{L}(b) .
$$

Therefore $i_{\kappa}$ is an intertwiner as required.

(2) The canonical map can $: H \otimes H \rightarrow H \otimes H$, can $: u \otimes v \mapsto u_{(1)} \otimes u_{(2)} v$ is a linear isomorphism. Consider the diagram

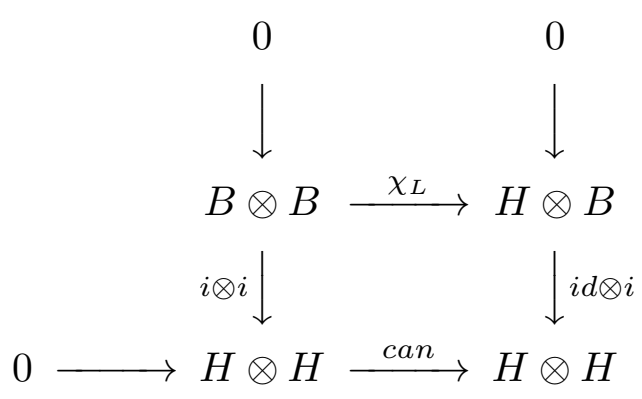

Clearly, both the rows and the columns of the diagram (2-1) are exact. Moreover for any $b, b^{\prime} \in B$

$$
(i d \otimes i) \circ \chi_{L}\left(b \otimes b^{\prime}\right)=b_{(1)} \otimes i\left(b_{(\infty)} b^{\prime}\right)=b_{(1)} \otimes i\left(b_{(\infty)}\right) i\left(b^{\prime}\right)
$$


and hence the diagram (2-1) is also commutative. Therefore we conclude that the sequence $0 \rightarrow B \otimes B \stackrel{\chi_{L}}{\longrightarrow} H \otimes B$ is exact, i.e. the map $\chi_{L}$ is injective.

Remark 2.3. The second assertion of Proposition 2.2., i.e., the injectiveness of $\chi_{L}$, is a dual version of the statement that the action of a group on its homogeneous space is transitive.

Proposition 2.4. Let $B$ be an embeddable $H$-space corresponding to the character $\kappa: B \rightarrow k$. Define a right ideal $J_{\kappa} \subset H$ by $J_{\kappa}=\left\{\sum_{j}\left(i_{\kappa}\left(b_{j}\right)-\kappa\left(b_{j}\right)\right) u_{j} ; \forall b_{j} \in\right.$ $\left.B, \forall u_{j} \in H\right\}$. Then $J_{\kappa}$ is an $H$-coideal.

Proof. Clearly

$$
\epsilon\left(i_{\kappa}(b)-\kappa(b)\right)=\epsilon\left(b_{(1)}\right) \kappa\left(b_{(\infty)}\right)-\kappa(b)=\kappa(b)-\kappa(b)=0 .
$$

Furthermore

$$
\begin{aligned}
\Delta\left(i_{\kappa}(b)-\kappa(b)\right) & =i_{\kappa}(b)_{(1)} \otimes i_{\kappa}(b)_{(2)}-\kappa(b) 1 \otimes 1 \\
& =b_{(1)} \otimes\left(i_{\kappa}\left(b_{(\infty)}\right)-\kappa\left(b_{(\infty)}\right)\right)+b_{(1)} \kappa\left(b_{(\infty)}\right) \otimes 1-\kappa(b) 1 \otimes 1 \\
& =b_{(1)} \otimes\left(i_{\kappa}\left(b_{(\infty)}\right)-\kappa\left(b_{(\infty)}\right)\right)+\left(i_{\kappa}(b)-\kappa(b)\right) \otimes 1 .
\end{aligned}
$$

Therefore for any $b \in B$,

$$
\Delta\left(i_{\kappa}(b)-\kappa(b)\right) \in H \otimes J_{\kappa} \oplus J_{\kappa} \otimes H
$$

so that $J_{\kappa}$ is a coideal as stated.

Since $J_{\kappa}$ is a coideal of $H$, the vector space $C=H / J_{\kappa}$ is a coalgebra and the canonical projection $\pi: H \rightarrow C$ is a coalgebra map. This in turn implies that $H$ is a right $C$-comodule with the coaction $\Delta_{R}=(i d \otimes \pi) \circ \Delta: H \rightarrow H \otimes C$. Let $H^{c o C}=\left\{u \in H ; \Delta_{R} u=u \otimes \pi(1)\right\}$.

Proposition 2.5. Let $B$ be an embeddable $H$-space corresponding to the character $\kappa: B \rightarrow k, J_{\kappa}$ be as in Proposition 2.4 and $C=H / J_{\kappa}$. Then:

(1) $H^{c o C}$ is a subalgebra of $H$.

(2) $B$ is a subalgebra of $H^{c o C}$.

Proof. (1) Since ker $\pi=J_{\kappa}$ is a right ideal in $H$ there is a natural right action $\rho_{0}: C \otimes H \rightarrow C$ of $H$ on $C$ given by the commutative diagram

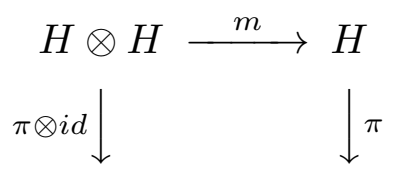


In other words for any $a \in C$ and $u \in H, \rho_{0}(a, u)=\pi(v u)$, where $v \in \pi^{-1}(a)$. For any $u, v \in H^{c o C}$ we compute

$$
\begin{aligned}
\Delta_{R}(u v) & =u_{(1)} v_{(1)} \otimes \pi\left(u_{(2)} v_{(2)}\right) \\
& =u(1) v_{(1)} \otimes \rho_{0}\left(\pi\left(u_{(2)}\right), v_{(2)}\right) \\
& =u v_{(1)} \otimes \rho_{0}\left(\pi(1), v_{(2)}\right) \\
& =u v_{(1)} \otimes \pi\left(v_{(2)}\right)=u v \otimes \pi(1) .
\end{aligned}
$$

Therefore $u v \in H^{c o C}$ and $H^{c o C}$ is a subalgebra of $H$ as required.

(2) For any $b \in B$ we compute

$$
\begin{aligned}
\Delta_{R}\left(i_{\kappa}(b)\right) & =i_{\kappa}(b)_{(1)} \otimes \pi\left(i_{\kappa}(b)_{(2)}\right)=b_{(1)} \otimes \pi\left(i_{\kappa}\left(b_{(\infty)}\right)\right) \\
& =b_{(1)} \otimes \kappa\left(b_{(\infty)}\right) \pi(1)=i_{\kappa}(b) \otimes \pi(1) .
\end{aligned}
$$

Hence $i_{\kappa}: B \hookrightarrow H^{c o C}$ is the required algebra inclusion.

Proposition 2.5. shows therefore that if $H^{c o C} \subset i_{\kappa}(B)$ then the embeddable $H$-space $B$ may be identified with the quantum quotient space $H^{c o C}$.

\section{A POSSIBLE GENERALISATION OF QUANTUM PRINCIPAL BUNDLES}

Once an $H$-embeddable space $B$ is identified with a quotient space $H^{c o C}$, it is natural to view $H$ as a total space of a principal bundle over $B$. Therefore one would like to apply the general theory of quantum principal bundles of $[\mathrm{BM}]$ to this case too. In general, however, neither $C$ is a Hopf algebra nor, if it happens to be a Hopf algebra, $C$ is a quantum subgroup of $H$. Hence the induced coaction of $C$ on $H$ is not an algebra map. Therefore to develop a gauge theory on embeddable homogeneous spaces one needs to generalise the theory of quantum principal bundles. In this section we propose such a generalisation. It is based on a simple observation that the structure of quantum principal bundles is mainly determined by the coalgebra structure of the quantum group. The algebra structure enters in a few places and it is really needed when the covariance properties are discussed (for example we need an antipode to analyse the transformation properties of a connection).

Let $C$ be a coalgebra and let $P$ be an algebra and a right $C$-comodule. Assume that there is an action $\rho: P \otimes C \otimes P \rightarrow P \otimes C$ of $P$ on $P \otimes C$ and an element $1 \in C$ such that 
(2) The following diagram

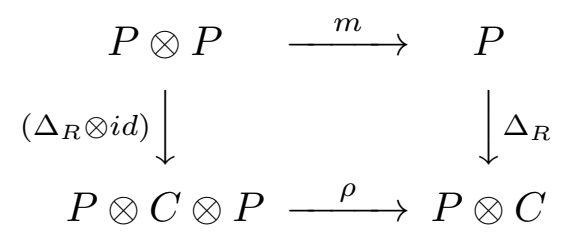

where $m$ is a product in $P$, is commutative.

Define $B=P^{c o C}=\left\{u \in P ; \Delta_{R} u=u \otimes 1\right\}$.

Lemma 3.1. $B$ is a subalgebra of $P$.

Proof. Take any $u, v \in B$. Then

$$
\Delta_{R}(u v)=\rho\left(u_{(0)} \otimes u_{(1)}, v\right)=\rho(u \otimes 1, v)=u v_{(0)} \otimes v_{(1)}=u v \otimes 1
$$

Definition 3.2. Let $P, C, \rho$ and $B$ be as before. We say that $P(B, C, \rho)$ is a $C$-Galois extension or a quantum $\rho$-principal bundle (with universal differential structure) if the canonical map $\chi: P \otimes_{B} P \rightarrow P \otimes C, \chi: u \otimes_{B} v \mapsto u v_{(0)} \otimes v_{(1)}$ is a bijection.

Example 3.3. A quantum principal bundle $P(B, H)$ as defined in $[\mathrm{BM}]$ is a $\rho$ principal bundle with the action $\rho: P \otimes H \otimes P \rightarrow P \otimes H$ given by $\rho(u \otimes a, v)=$ $u v_{(0)} \otimes a v_{(1)}$

Example 3.4. Let $H$ be a Hopf algebra, $C$ a coalgebra and $\pi: H \rightarrow C$ a coalgebra projection. Then $H$ is a right $C$-comodule with a coaction $\Delta_{R}=(i d \otimes \pi) \circ \Delta$. Denote $1=\pi(1) \in C$ and define $B=H^{c o C}$ as before. Assume that $\operatorname{ker} \pi$ is a minimal right ideal in $H$ such that $\{u-\epsilon(u) ; u \in B\} \subset \operatorname{ker} \pi$ (compare Section 2). Then we can define a canonical right action $\rho_{0}: C \otimes H \rightarrow C$ as in the proof of Proposition 2.5. Furthermore we define

$$
\rho(u \otimes a, v)=u v_{(1)} \otimes \rho_{0}\left(a, v_{(2)}\right)
$$

for any $u, v \in H, a \in C$. With these definitions $H(B, C, \rho)$ is a quantum $\rho$-principal bundle.

Proof. First we need to show that $\rho: H \otimes C \otimes H \rightarrow H \otimes C$ is a right action and it has the properties (1) and (2). Since $\rho_{0}$ is a right action, for any $u, v, w \in H$, $a \in C$ we compute

$$
\rho(u \otimes a, v w)=u v_{(1)} w_{(1)} \otimes \rho_{0}\left(a, v_{(2)} w_{(2)}\right)=u v_{(1)} w_{(1)} \otimes \rho_{0}\left(\rho_{0}\left(a, v_{(2)}\right), w_{(2)}\right)
$$


and thus $\rho$ is an action as required. Furthermore

$$
\rho(u \otimes 1, v)=u v_{(1)} \otimes \rho_{0}\left(1, v_{(2)}\right)=u v_{(1)} \otimes \pi\left(v_{(2)}\right)=u v_{(0)} \otimes v_{(1)},
$$

and

$$
\rho\left(u_{(0)} \otimes u_{(1)}, v\right)=u_{(1)} v_{(1)} \otimes \rho_{0}\left(\pi\left(u_{(2)}\right), v_{(2)}\right)=u_{(1)} v_{(1)} \otimes \pi\left(u_{(2)} v_{(2)}\right)=\Delta_{R}(u v) .
$$

Therefore $\rho$ has all the required properties.

To prove that the canonical map $\chi$ is bijective we first note that, by assumption, $\operatorname{ker} \pi \subset m \circ\left(\left.\operatorname{ker} \pi\right|_{B} \otimes H\right)$ and then use a suitably modified argument of the proof of Lemma 5.2. of $[\mathrm{BM}]$ to deduce that $\chi$ is a bijection. It is clear that $\chi$ is a surjection since for any $\sum_{k} u_{k} \otimes a_{k} \in H \otimes C$ we can choose $\sum_{k} u_{k} S v_{k(1)} \otimes_{B} v_{k(2)} \in H \otimes_{B} H$, where $\forall k, v_{k} \in \pi^{-1}\left(a_{k}\right)$, and compute

$$
\begin{aligned}
\chi\left(\sum_{k} u_{k} S v_{k(1)} \otimes_{B} v_{k(2)}\right) & =\sum_{k} u_{k}\left(S v_{k(1)}\right) v_{k(2)} \otimes \pi\left(v_{k(3)}\right) \\
& =\sum_{k} u_{k} \otimes \pi\left(v_{k}\right)=\sum_{k} u_{k} \otimes a_{k} .
\end{aligned}
$$

Next we compute $\operatorname{ker} \chi \subset H \otimes_{B} H$. Take any $\sum_{k} u_{k} \otimes_{B} v_{k} \in \operatorname{ker} \chi$. Then $\sum_{k} u_{k} v_{k(1)} \otimes \pi\left(v_{k(2)}\right)=0$. Applying $i d \otimes \epsilon$ to the last equality we then find that $\sum_{k} u_{k} v_{k}=0$, i.e., $\sum_{k} u_{k} \otimes v_{k} \in \operatorname{ker} m$. Any $\sum_{i} w_{i}^{\prime} \otimes w_{i}^{\prime \prime} \in \operatorname{ker} m$ can be written as $\sum_{k} u_{k} S v_{k(1)} \otimes v_{k(2)} \in H \otimes H$, where $\forall k, v_{k} \in$ ker $\epsilon$ and $u_{k}$ are linearly independent. Thus

$$
\chi\left(\sum_{i} w_{i}^{\prime} \otimes_{B} w_{i}^{\prime \prime}\right)=\chi\left(\sum_{k} u_{k} S v_{k(1)} \otimes_{B} v_{k(2)}\right)=\sum_{k} u_{k} \otimes \pi\left(v_{k}\right) .
$$

If $\sum_{i} w_{i}^{\prime} \otimes_{B} w_{i}^{\prime \prime} \in \operatorname{ker} \chi$ then $\sum_{k} u_{k} \otimes \pi\left(v_{k}\right)=0$, thus for all $k, \pi\left(v_{k}\right)=0$. By assumption $v_{k}=\sum_{j} b_{k}^{j} v_{k}^{j}$, where $\left.b_{k}^{j} \in \operatorname{ker} \epsilon\right|_{B}=\left.\operatorname{ker} \pi\right|_{B}$. Therefore

$$
\begin{aligned}
\sum_{i} w_{i}^{\prime} \otimes_{B} w_{i}^{\prime \prime} & =\sum_{k} u_{k} S v_{k(1)} \otimes_{B} v_{k(2)}=\sum_{j, k} u_{k}\left(S v_{k(1)}^{j}\right) S b_{k(1)}^{j} \otimes_{B} b_{k(2)}^{j} v_{k(2)}^{j} \\
& =\sum_{j, k} \epsilon\left(b_{k}^{j}\right) u_{k} S v_{k(1)}^{j} \otimes_{B} v_{k(2)}^{j}=0
\end{aligned}
$$

So $\operatorname{ker} \chi=0$, and $\chi$ is a bijection as required.

Therefore we have shown that an embeddable $H$ space which is a quotient space $B=H^{c o C}$ as described in Section 2 may be indentified with a base manifold of the generalised quantum principal bundle, or equivalently that $H$ is a $C$-Galois 


\section{Manin's Plane AS A QUANTUM QUOTIEnt SPACE}

In this section we show that Manin's plane is a quotient space of the quantum general linear group $G L_{q}(2, \mathbf{C})$. Recall that Manin's plane $\mathbf{C}_{q}^{2}$ is defined for any non-zero $q \in \mathbf{C}$ as an associative polynomial algebra over $\mathbf{C}$ generated by $1, x, y$ subject to the relations $x y=q y x$. It is a quantum homogeneous space of the quantum linear group $G L_{q}(2, \mathbf{C}) . G L_{q}(2, \mathbf{C})$ is defined as follows. First we consider an algebra generated by the matrix $\mathbf{t}=\left(\begin{array}{ll}\alpha & \beta \\ \gamma & \delta\end{array}\right)$ and the relations

$$
\begin{gathered}
\alpha \beta=q \beta \alpha, \quad \alpha \gamma=q \gamma \alpha, \quad \alpha \delta=\delta \alpha+\left(q-q^{-1}\right) \beta \gamma, \\
\beta \gamma=\gamma \beta, \quad \beta \delta=\delta \beta, \quad \gamma \delta=q \delta \gamma .
\end{gathered}
$$

The quantum determinant $c=\alpha \delta-q \beta \gamma$ is central in the algebra (4-1) thus we enlarge it with $c^{-1}$ and call the resulting algebra $G L_{q}(2, \mathbf{C})$. The quantum linear group $G L_{q}(2, \mathbf{C})$ is a Hopf algebra of a matrix group type, i.e.

$$
\Delta \mathbf{t}=\mathbf{t} \dot{\otimes} \mathbf{t}, \quad \epsilon \mathbf{t}=1, \quad S \mathbf{t}=c^{-1}\left(\begin{array}{cc}
\delta & -q^{-1} \beta \\
-q \gamma & \alpha
\end{array}\right) .
$$

The left coaction of $G L_{q}(2, \mathbf{C})$ on $\mathbf{C}_{q}^{2}$ is given by

$$
\Delta_{L}\left(\begin{array}{l}
x \\
y
\end{array}\right)=\left(\begin{array}{ll}
\alpha & \beta \\
\gamma & \delta
\end{array}\right) \dot{\otimes}\left(\begin{array}{l}
x \\
y
\end{array}\right) \text {. }
$$

$\mathbf{C}_{q}^{2}$ in not only a homogeneous space of $G L_{q}(2, \mathbf{C})$ but also it is an embeddable $G L_{q}(2, \mathbf{C})$-space. The linear map $\kappa: \mathbf{C}_{q}^{2} \rightarrow \mathbf{C}, \kappa\left(x^{n} y^{m}\right)=\delta_{m 0}, m, n \in \mathbf{Z}_{\geq 0}$ is a character of $\mathbf{C}_{q}^{2}$. By Proposition 2.2. it induces an algebra map $i_{\kappa}: \mathbf{C}_{q}^{2} \rightarrow$ $G L_{q}(2, \mathbf{C})$, which is explicitly given by $i_{\kappa}(x)=\alpha, i_{\kappa}(y)=\gamma$. The map $i_{\kappa}$ is clearly an inclusion. Thus the right ideal $J_{\kappa}$ is generated by $\alpha-1$ and $\gamma$. The coalgebra $C=G L_{q}(2, \mathbf{C}) / J_{\kappa}$ may be easily computed. It is spanned by $a_{m, n}=\pi\left(\beta^{m} c^{n}\right)$, $m \in \mathbf{Z}_{>0}, n \in \mathbf{Z}$ and $a_{0,0}=1=\pi(1)$, where $\pi: G L_{q}(2, \mathbf{C}) \rightarrow C$ is a canonical surjection. To see that the $a_{m, n}$ really span $C$ we note that since $J_{\kappa}$ is generated by $\alpha-1$ and $\gamma$ as a right ideal in $G L_{q}(2, \mathbf{C})$, every $\alpha$ which multiplies any element of $G L_{q}(2, \mathbf{C})$ from the left is replaced by 1 and similarly any $\gamma$ is replaced by 0 when the resulting element of $G L_{q}(2, \mathbf{C})$ is acted upon by $\pi$. Then we compute

$$
\begin{aligned}
\pi\left(\alpha^{k} \beta^{l} \gamma^{m} \delta^{n} c^{r}\right) & =\pi\left(\beta^{l} \gamma^{m} \delta^{n} c^{r}\right) \\
& =\delta_{m 0} \pi\left(\beta^{l} \delta^{n} c^{r}\right)=\delta_{m 0} q^{l n} \pi\left(\delta^{n} \beta^{l} c^{r}\right) \\
& =\delta_{m 0} q^{l n}\left(\pi\left(\alpha \delta \delta^{n-1} \beta^{l} c^{r}\right)-q^{-1} \pi\left(\gamma \beta \delta^{n-1} \beta^{l} c^{r}\right)\right)
\end{aligned}
$$


Therefore any element of $C=\pi\left(G L_{q}(2, \mathbf{C})\right)$ may be expressed as a linear combination of $a_{m, n}$.

The coalgebra structure of $C$ is found from the coalgebra structure of $G L_{q}(2, \mathbf{C})$, since $\Delta_{C}=(\pi \otimes \pi) \circ \Delta_{G L_{q}(2, \mathbf{C})}$. Explicitly

$$
\Delta a_{m, n}=\sum_{k=0}^{m}\left(\begin{array}{c}
m \\
k
\end{array}\right)_{q} a_{k, n} \otimes a_{m-k, n+k}, \quad \epsilon\left(a_{m, n}\right)=\delta_{m 0}
$$

where the quantum binomial coefficients are defined by

$$
\left(\begin{array}{c}
m \\
k
\end{array}\right)_{q}=\frac{[m]_{q} !}{[m-k]_{q} ![k]_{q} !}, \quad[m]_{q}=\frac{q^{m}-q^{-m}}{q-q^{-1}}, \quad[m]_{q} !=\prod_{k=1}^{m}[k]_{q}, \quad[0]_{q} !=1 .
$$

The next step in the identification of $\mathbf{C}_{q}^{2}$ as a quantum quotient space consists of computing the fixed point subalgebra $B=G L_{q}(2, \mathbf{C})^{c o C}$. For a general monomial $\alpha^{k} \gamma^{l} \beta^{m} \delta^{n} c^{r} \in G L_{q}(2, \mathbf{C}), k, l, m, n \in \mathbf{Z}_{\geq 0}, r \in \mathbf{Z}$, we find

$$
\begin{aligned}
\Delta_{R}\left(\alpha^{k} \gamma^{l} \beta^{m} \delta^{n} c^{r}\right) & =\alpha^{k} \gamma^{l} c^{r} \sum_{i=0}^{m} \sum_{j=0}^{n} q^{j(m-i)}\left(\begin{array}{c}
m \\
i
\end{array}\right)_{q}\left(\begin{array}{c}
n \\
j
\end{array}\right)_{q} \\
& \times \alpha^{m-i} \beta^{i} \gamma^{n-j} \delta^{j} \otimes a_{m+n-(i+j), i+j+r}
\end{aligned}
$$

The right hand side of (4-3) has the form $u \otimes 1$ for some $u \in G L_{q}(2, \mathbf{C})$ if and only if $m=n=r=0$. Thus $B$ is spanned by all $\alpha^{k} \gamma^{l}$. Therefore $B \subset i_{\kappa}\left(\mathbf{C}_{q}^{2}\right)$ and since $i_{\kappa}\left(\mathbf{C}_{q}^{2}\right) \subset B$ by Proposition 2.5 we conclude that $\mathbf{C}_{q}^{2} \cong G L_{q}(2, \mathbf{C})^{c o C}$. By Example $3.4 G L_{q}(2, \mathbf{C})\left(\mathbf{C}_{q}^{2}, C, \rho\right)$ is a quantum principal $\rho$-bundle. The action $\rho_{0}: C \otimes G L_{q}(2, \mathbf{C}) \rightarrow C$ is given explicitly by

$$
\rho_{0}\left(a_{i, j}, \alpha^{k} \beta^{l} \gamma^{m} \delta^{n} c^{r}\right)=\delta_{m 0} q^{i(n-k)+l n} a_{i+l, j+n+r}
$$

We can now proceed to define an algebra structure on $C$ so that it becomes a Hopf algebra. We define the product in $C$ by

$$
a_{k, l} a_{m, n}=q^{l m-k n} a_{k+m, l+n}
$$

First we notice that $a_{0,0}=1$ is the unit element with respect to this product. Next 
compute

$$
\begin{aligned}
\Delta\left(a_{k, l}\right) \Delta\left(a_{m, n}\right) & =\sum_{i=0}^{k} \sum_{j=0}^{m}\left(\begin{array}{c}
k \\
i
\end{array}\right)_{q}\left(\begin{array}{c}
m \\
j
\end{array}\right)_{q} a_{i, l} a_{j, n} \otimes a_{k-i, l+i} a_{m-j, n+j} \\
& =q^{l m-n k} \sum_{i=0}^{k} \sum_{j=0}^{n} q^{i m-k j}\left(\begin{array}{c}
k \\
i
\end{array}\right)_{q}\left(\begin{array}{c}
m \\
j
\end{array}\right)_{q} a_{i+j, l+n} \otimes a_{k+m-(i+j), l+n+i+j} \\
& =q^{l m-n k} \sum_{r=0}^{k+m}\left(\begin{array}{c}
k+m \\
i
\end{array}\right)_{q} a_{r, l+n} \otimes a_{k+m-r, l+n+r} \\
& =q^{l m-k n} \Delta\left(a_{k+m, l+n}\right)=\Delta\left(a_{k, l} a_{m, n}\right) .
\end{aligned}
$$

The third equality is a consequence of the following property of the $q$-deformed binomial coefficients

$$
\forall r \in[0, k+m], \quad \sum_{i=0}^{k} \sum_{j=0}^{n} q^{i m-k j}\left(\begin{array}{c}
k \\
i
\end{array}\right)_{q}\left(\begin{array}{c}
m \\
j
\end{array}\right)_{q}=\left(\begin{array}{c}
k+m \\
r
\end{array}\right)_{q} .
$$

Clearly the counit of $C$ is an algebra homomorphism. Before we define an antipode we show that $C$ is a polynomial algebra. Let $a=a_{0,1}, a^{-1}=a_{0,-1}, b=a_{1,0}$. Then for any $m \in \mathbf{Z}_{\geq 0}, n \in \mathbf{Z}$,

$$
a_{m, n}=q^{-m n} a^{n} b^{m}, \quad a b=q^{2} b a, \quad a a^{-1}=a^{-1} a=1 .
$$

Therefore $C$ is a polynomial algebra indeed, and it is isomorphic to $\mathbf{C}_{q^{2}}^{2}\left[x^{-1}\right]$. The coalgebra structure of $C$ written in terms of $a$ and $b$ reads

$$
\Delta a^{ \pm 1}=a^{ \pm 1} \otimes a^{ \pm 1}, \quad \Delta b=1 \otimes b+b \otimes a, \quad \epsilon\left(a^{ \pm 1}\right)=1, \quad \epsilon(b)=0
$$

and hence the antipode is defined as $S a^{ \pm 1}=a^{\mp 1}, S b=-b a^{-1}$.

We have just shown that $C$ may be equipped with an algebra structure of $\mathbf{C}_{q^{2}}^{2}\left[x^{-1}\right]$, and then the coalgebra structure of $C$ becomes a standard coalgebra structure of the latter. Therefore we have proven

\section{Theorem 4.1.}

$$
\mathbf{C}_{q}^{2}=G L_{q}(2, \mathbf{C})^{c o \mathbf{C}_{q^{2}}^{2}\left[x^{-1}\right]} .
$$

Notice that clearly neither $\pi: G L_{q}(2, \mathbf{C}) \rightarrow \mathbf{C}_{q^{2}}^{2}\left[x^{-1}\right]$ nor $\Delta_{R}=(i d \otimes \pi) \circ \Delta:$ $G L_{q}(2, \mathbf{C}) \rightarrow G L_{q}(2, \mathbf{C}) \otimes \mathbf{C}_{q^{2}}^{2}\left[x^{-1}\right]$ are algebra maps. Still, following the proposal of Section 3 we can analyse the generalised principal bundle $G L_{q}(2, \mathbf{C})\left(\mathbf{C}_{q}^{2}, \mathbf{C}_{q^{2}}^{2}\left[x^{-1}\right], \rho, \pi\right)$. In particular we can truly develop a gauge theory, define connections and their cur- 


\section{Podleś' SPhere as a quantum Quotient SPACE}

In this section we prove that the quantum two-sphere is a quantum quotient space in the sense expalined in Section 2. In our presentation of the quantum sphere we follow the conventions of $[\mathrm{NM}]$.

The general quantum two-sphere $S_{q}^{2}(\mu, \nu)$ is a polynomial algebra generated by the unit and $x, y, z$, and the relations

$$
\begin{aligned}
& x z=q^{2} z x, \quad x y=-q(\mu-z)(\nu+z), \\
& y z=q^{-2} z y, \quad y x=-q\left(\mu-q^{-2} z\right)\left(\nu+q^{-2} z\right),
\end{aligned}
$$

where $\mu, \nu$ and $q \neq 0$ are real parameters, $\mu \nu \geq 0,(\mu, \nu) \neq(0,0)$. The quantum sphere is a $*$-algebra with the $*$-structure $x^{*}=-q y, z^{*}=z$.

The quantum sphere $S_{q}^{2}(\mu, \nu)$ is an $S U_{q}(2)$ homogeneous quantum space. $S U_{q}(2)$ is defined as a quotient of $G L_{q}(2, \mathbf{C})$ by the relation $c=1$, and has a $*$-structure given by $\delta=\alpha^{*}, \gamma=-q^{-1} \beta^{*}$. The coaction of $S U_{q}(2)$ on $S_{q}^{2}(\mu, \nu)$ is defined as follows. Let $\phi_{-}=x, \phi_{0}=\left(1+q^{-2}\right)^{-1 / 2}\left(\mu-\nu-\left(1+q^{-2}\right) z\right), \phi_{+}=y$. Then

$$
\Delta_{L}\left(\begin{array}{c}
\phi_{-} \\
\phi_{0} \\
\phi_{+}
\end{array}\right)=\left(\begin{array}{ccc}
\alpha^{2} & \left(1+q^{-2}\right)^{1 / 2} \alpha \beta & \beta^{2} \\
\left(1+q^{-2}\right)^{1 / 2} \alpha \gamma & 1+\left(q+q^{-1}\right) \beta \gamma & \left(1+q^{-2}\right)^{1 / 2} \beta \delta \\
\gamma^{2} & \left(1+q^{-2}\right)^{1 / 2} \gamma \delta & \delta^{2}
\end{array}\right) \dot{\otimes}\left(\begin{array}{c}
\phi_{-} \\
\phi_{0} \\
\phi_{+}
\end{array}\right) .
$$

The quantum sphere $S_{q}^{2}(\mu, \nu)$ is not only a quantum homogeneous space but also it is an embeddable $S U_{q}(2)$-space. There is a $*$-character $\kappa: S_{q}^{2}(\mu, \nu) \rightarrow \mathbf{C}$ given by

$$
\kappa(x)=q \sqrt{\mu \nu}, \quad \kappa(y)=-\sqrt{\mu \nu}, \quad \kappa(z)=0 .
$$

Therefore there is also a $*$-algebra homomorphism $i_{\kappa}: S_{q}^{2}(\mu, \nu) \rightarrow S U_{q}(2)$, which reads explicitly

$$
\begin{gathered}
i_{\kappa}(x)=\sqrt{\mu \nu}\left(q \alpha^{2}-\beta^{2}\right)+(\mu-\nu) \alpha \beta, \\
i_{\kappa}(y)=\sqrt{\mu \nu}\left(q \gamma^{2}-\delta^{2}\right)+(\mu-\nu) \gamma \delta, \\
i_{\kappa}(z)=-\sqrt{\mu \nu}(q \alpha \gamma-\beta \delta)-(\mu-\nu) \beta \gamma,
\end{gathered}
$$

and is clearly an inclusion. From now on we assume that $\mu \neq \nu$ (but see also Remark 5.5). In this case $S_{q}^{2}(\mu, \nu)$ depends on two real parameters only, namely $q$ and $p=\frac{\sqrt{\mu \nu}}{\mu-\nu}$. By Proposition 2.4. the inclusion $i_{\kappa}$ induces a coideal $J_{\kappa} \subset S U_{q}(2)$, generated as a right ideal in $S U_{q}(2)$ by the following three elements 
Therefore we can construct the coalgebra $C(p)=S U_{q}(2) / J_{\kappa}$, and the corresponding quotient space $B(p)=S U_{q}(2)^{c o C(p)}$ as described in Section 2. At the end of this procedure we identify $B(p)$ with $S_{q}^{2}(\mu, \nu), \mu \neq \nu$. We start with the coalgebra $C(p)$.

Proposition 5.1. $C(p)$ is a vector space spanned by $1=\pi(1), x_{n}=\pi\left(\alpha^{n}\right)$ and $y_{n}=\pi\left(\delta^{n}\right)$, where $\pi: S U_{q}(2) \rightarrow C(p)$ is a canonical surjection and $n \in \mathbf{Z}_{>0}$.

Proof. For any $u \in S U_{q}(2)$ we use the explicit form of the generators of $J_{\kappa}$, and the relations in $S U_{q}(2)$ to find that

$$
\begin{aligned}
\pi(\beta u) & =\pi(\beta \alpha \delta u)-q \pi(\beta \gamma \beta u) \\
& =q^{-1} \pi(\alpha \beta \delta u)-q \pi(\beta \gamma \beta u) \\
& =-p \pi\left(\alpha^{2} \delta u\right)+p q^{-1} \pi\left(\beta^{2} \delta u\right) \\
& +p \pi(\delta u)+p q \pi(\alpha \gamma \beta u)-p \pi(\beta \delta \beta u) \\
& =p \pi(\delta u)-p \pi(\alpha u),
\end{aligned}
$$

and similarly

$$
\pi(\gamma u)=p \pi(\delta u)-p \pi(\alpha u)
$$

From (5-1) it follows that for any $u \in S U_{q}(2), \pi\left(u \beta^{m} \gamma^{n}\right)=\pi\left(u \beta^{m+n}\right)$. Since $S U_{q}(2)$ is spanned by the monomials $\alpha^{m} \beta^{k} \gamma^{l}, \delta^{m} \beta^{k} \gamma^{l}$ (cf. Lemma 7.1.2 of [CP]) it suffices to prove that the following elements of $C(p)$,

$$
a_{k-}^{(n)}=\pi\left(\delta^{k} \beta^{n-k}\right), \quad a_{k+}^{(n)}=\pi\left(\alpha^{k} \beta^{n-k}\right),
$$

where $n \in \mathbf{Z}_{>0}, k=0,1, \ldots, n$, can be expressed as linear combinations of $1, x_{m}$, $y_{m}$. Clearly $a_{0-}^{(n)}=a_{0+}^{(n)}$. Thus we simply write $a_{0}^{(n)}$. Also, $a_{n+}^{(n)}=x_{n}$ and $a_{n-}^{(n)}=y_{n}$. For $n=1, a_{0}^{(1)}=\pi(\beta)=p\left(y_{1}-x_{1}\right)$. For a general $n$ we apply the rules (5-1) to $a_{k \pm}^{(n)}$ and we express the latter in terms of $a_{l \pm}^{(m)}, m<n$ and $x_{n}, y_{n}$. We make the inductive assumption that for all $m<n, a_{l \pm}^{(m)}$ can be written as linear combinations of $1, x_{r}, y_{r}$. Therefore, for $n \geq 2$ we arrive at the system of equations

$$
\begin{aligned}
& a_{k \pm}^{(n)} \pm p q^{ \pm k} a_{k+1 \pm}^{(n)} \mp p q^{\mp(k-1)} a_{k-1 \pm}^{(n)}= \pm p q^{ \pm k} a_{k-1 \pm}^{(n-2)}, \\
& a_{0}^{(n)}-p a_{1-}^{(n)}+p a_{1+}^{(n)}=0
\end{aligned}
$$

where $k=1,2, \ldots, n-1$. This is a system of $2 n-1$ equations with $2 n-1$ unknowns 
Obviously it has a solution if its determinant is non-zero. The determinant $D_{n}$ of the system (5-3) may be easily computed. It does not depend on $q$ and, by the Laplace theorem, it can be reduced to the determinant of the following $2 n-1 \times 2 n-1$ matrix

$$
\left(\begin{array}{cccccccccccc}
1 & -p & p & 0 & 0 & 0 & \ldots & 0 & 0 & 0 & 0 & 0 \\
p & 1 & 0 & -p & 0 & 0 & \ldots & 0 & 0 & 0 & 0 & 0 \\
-p & 0 & 1 & 0 & p & 0 & \ldots & 0 & 0 & 0 & 0 & 0 \\
0 & p & 0 & 1 & 0 & -p & \ldots & 0 & 0 & 0 & 0 & 0 \\
\ldots \ldots & \ldots & \ldots & \ldots & \ldots & \ldots & \ldots \ldots & \ldots & \ldots & \ldots & \ldots \\
0 & 0 & 0 & 0 & 0 & 0 & \ldots & 0 & 1 & 0 & -p & 0 \\
0 & 0 & 0 & 0 & 0 & 0 & \ldots & -p & 0 & 1 & 0 & p \\
0 & 0 & 0 & 0 & 0 & 0 & \ldots & 0 & p & 0 & 1 & 0 \\
0 & 0 & 0 & 0 & 0 & 0 & \ldots & 0 & 0 & -p & 0 & 1
\end{array}\right)
$$

Again by the Laplace theorem $D_{n}$ can be further developed to give

$$
D_{n}=A_{2 n-2}+p^{2}\left(A_{2 n-3}+A_{2 n-4}\right)+p^{4} A_{2 n-5},
$$

where $A_{m}$ is zero for negative $m, A_{0}=1$ and for any $m<2 n-1, A_{m}$ is the determinant of the matrix obtained from (5-4) by removing first $2 n-1-m$ rows and columns. The determinants $A_{m}$ are the standard ones and we finally obtain the determinant of the system (5-3) as a polynomial

$$
D_{n}=P_{n-1}\left(p^{2}\right) \equiv \sum_{k=0}^{n-1}\left(\begin{array}{c}
2 n-1-k \\
k
\end{array}\right) p^{2 k}
$$

For any $x \in \mathbf{R}_{\geq 0}, P_{n}(x) \geq 1$, and hence $D_{n} \neq 0$ for any real $p$. Therefore the system (5-3) always has a solution and the coalgebra $C(p)$ is spanned by $x_{n}, y_{n}$, $n \in \mathbf{Z}_{>0}$ and 1 as required.

The vector space $C(p)$ has a coalgebra structure induced by $\pi$ from the coalgebra structure of $S U_{q}(2)$. The coproduct reads explicitly

$$
\Delta x_{n}=\sum_{k=0}^{n-1} q^{-(n-k) k}\left(\begin{array}{l}
n \\
k
\end{array}\right)_{q} a_{k+}^{(n)} \otimes a_{k+}^{(n)}, \quad \Delta y_{n}=\sum_{k=0}^{n-1} q^{(n-k) k}\left(\begin{array}{l}
n \\
k
\end{array}\right)_{q} a_{k-}^{(n)} \otimes a_{k-}^{(n)}
$$

where $a_{k \pm}^{(n)}$ are given by (5-2). Therefore the coalgebra $C(p)$ is cocommutative.

Remark 5.2. It is an interesting problem, whether it is possible to define a Hopf algebra structure on $C(p)$. For example, for $n=1$ we have

$$
\Delta x_{1}=\left(1+p^{2}\right) x_{1} \otimes x_{1}-p^{2}\left(x_{1} \otimes y_{1}+y_{1} \otimes x_{1}-y_{1} \otimes y_{1}\right) \text {, }
$$


If we define

$$
x_{1}^{\prime}=\frac{1}{\mu-\nu}\left(\mu x_{1}-\nu y_{1}\right), \quad y_{1}^{\prime}=\frac{1}{\mu-\nu}\left(\mu y_{1}-\nu x_{1}\right)
$$

then $x_{1}^{\prime}$ and $y_{1}^{\prime}$ are group-like, i.e. $\Delta x_{1}^{\prime}=x_{1}^{\prime} \otimes x_{1}^{\prime}$ and $\Delta y_{1}^{\prime}=y_{1}^{\prime} \otimes y_{1}^{\prime}$. If it were posible to define a new basis of $C(p)$ consisting only of group-like elements then clearly we would be able to solve the above problem and make $C(p)$ into a Hopf algebra of functions on $U(1)$.

Remark 5.3. According to [P1] quantum spheres can also be defined for a discrete series of complex numbers $p$ given by $p^{2}=-\left(q^{k}+q^{-k}\right)^{-2}, k=1,2, \ldots$ It is shown in [P2] that such quantum spheres are $*$-embeddable in $S U_{q}(2)$ for $k=1$.

One easily finds that $P_{n}(x-1 / 4)=\sum_{k=0}^{n} c_{k}^{n} x^{k}$, where

$$
c_{k}^{n}=\sum_{l=k}^{n}(-1 / 4)^{l-k}\left(\begin{array}{c}
2 n+1-l \\
l
\end{array}\right)\left(\begin{array}{l}
l \\
k
\end{array}\right)
$$

For any $n$ and any $0 \leq k \leq n, c_{k}^{n} \geq c_{0}^{n}=(n+1) / 4^{n}$ and thus all the coefficients $c_{k}^{n}$ are positive. Therefore $P_{n}(x-1 / 4) \neq 0$ for all real $x \geq 0$. This implies that the determinants $D_{n}$ of the proof of Proposition 5.1 are non-zero provided that $p^{2} \geq-1 / 4$. Since for any $q, q+q^{-1} \geq 2$ we see that the assertion of Proposition 5.1 holds for the exceptional quantum spheres too.

Propositon 5.4. Let $C(p)$ be a coalgebra described in Proposition 5.1 and let $B(p)=S U_{q}(2)^{\operatorname{coC}(p)}$. Then $i_{\kappa}\left(S_{q}^{2}(\mu, \nu)\right)=B(p)$ for all $\mu \neq \nu$ such that $p=\frac{\sqrt{\mu \nu}}{\mu-\nu}$.

Proof. By Proposition 2.5, $i_{\kappa}\left(S_{q}^{2}(\mu, \nu)\right) \subset B(p)$, therefore we need to show that $B(p) \subset i_{\kappa}\left(S_{q}^{2}(\mu, \nu)\right)$. We introduce the grading $d: S U_{q}(2) \rightarrow \mathbf{Z}$ by

$$
d(\alpha)=d(\beta)=1, \quad d(1)=0, \quad d(\gamma)=d(\delta)=-1, \quad d(u v)=d(u)+d(v),
$$

for any monomials $u, v \in S U_{q}(2)$. A set of all elements of $S U_{q}(2)$ of degree $k \in \mathbf{Z}$ forms a vector subspace of $S U_{q}(2)$, which we denote by $S U_{q}(2)^{(k)}$, and $S U_{q}(2)=$ $\bigoplus_{k \in \mathbf{Z}} S U_{q}(2)^{(k)}$. Moreover if $\Delta u=\sum_{i} u_{i}^{\prime} \otimes u_{i}^{\prime \prime}$ for any $u \in S U_{q}(2)^{(k)}$, then for all $i, d\left(u_{i}^{\prime}\right)=k$. To see that the last statement is true we can explicitly verify it for $\alpha$, $\beta, \gamma, \delta$ and then use definition (5-5) of $d$ to prove it for any $S U_{q}(2)$. Therefore $d$ induces a grading of $B(p)$ and $B(p)=\bigoplus_{k \in \mathbf{Z}} B(p)^{(k)}$.

Next we notice that $B(p)$ is contained in the subalgebra of $S U_{q}(2)$ spanned by 
To prove the required inclusion we observe that due to the form of $\pi$ and $C(p)$, $B(p)$ is a deformation of $B(0)$, i.e., $B(0)=\lim _{p \rightarrow 0} B(p)$. We denote by $B(p)_{2 n}^{(2 k)}$ the vector space of homogeneous polynomials $u \in B(p)$ of degree $2 n$ such that $d(u)=2 k,|k| \leq n$. Notice that $B(p)_{2 n}^{(2 k)}$ and $B(p)_{2 l}^{(2 k)}$ need not be distinct for $l \neq n . \quad B(0)_{2 n}^{(2 k)}$ is spanned by $\alpha^{m} \beta^{n+k-m} \gamma^{n-m} \delta^{m-k}$, where $m=k, k+1, \ldots, n$ for $k \geq 0$ and $m=0,1, \ldots, n+k$ for $k<0$, and hence is $n-|k|+1$-dimensional. This is exactly the dimension of $i_{\kappa}\left(S_{q}^{2}(\mu, \nu)\right)_{2 n}^{(2 k)}$. Suppose that $B(p)_{2 n}^{(2 k)}$ is at least $n-|k|+2$ dimensional. Then we can find $u \in B(p)_{2 n}^{(2 k)}$ that does not contain any of the monomials spanning $B(0)_{2 n}^{(2 k)}$. If $\lim _{p \rightarrow 0} u \neq 0$, then we would obtain that $B(0)_{2 n}^{(2 k)}$ is at least $n-|k|+2$ dimensional, hence contradiction. By $\lim _{p \rightarrow 0} u$ here we mean the polynomial obtained from $u$ by replacing its coefficients with their $p=0$ limits. Assume that $\lim _{p \rightarrow 0} u=0$. The polynomial $u$ may be written as a linear combination of monomials of degree $2 n$ with coefficients that vanish as polynomials when $p$ tends to 0 . Therefore there exists a positive integer $m$ such that $\lim _{p \rightarrow 0} p^{-m} u$ exists, is finite and non-zero, and is an element of $B(0)_{2 n}^{(2 k)}$. Thus we have a contradiction again. Since the above argument does not depend on $n$ and $k$, and $i_{\kappa}\left(S_{q}^{2}(\mu, \nu)\right) \subset B(p)$ we conclude that $i_{\kappa}\left(S_{q}^{2}(\mu, \nu)\right)=B(p)$.

Therefore we have shown that for $\mu \neq \nu$ the quantum sphere $S_{q}^{2}(\mu, \nu)$ is a quantum quotient space. By Example 3.4 we also have a principal $\rho$-bundle, $S U_{q}(2)\left(S_{q}^{2}(\mu, \nu), C(p), \rho, \pi\right)$.

Remark 5.5. When $\mu=\nu \neq 0$ the coideal $J_{\kappa}$ is generated as a right ideal in $S U_{q}(2)$ by the following elements

$$
q \alpha^{2}-\beta^{2}-q, \quad q \gamma^{2}-\delta^{2}+1, \quad q \alpha \gamma-\beta \delta .
$$

Therefore for any $u \in S U_{q}(2)$,

$$
\pi(\delta u)=\pi(\alpha u), \quad \pi(\beta u)=\pi(\gamma u), \quad \pi\left(\gamma^{2} u\right)=q \pi\left(\alpha^{2} u\right)-q \pi(u),
$$

and hence the coalgebra $C=S U_{q}(2) / J_{\kappa}$ is spanned by $1=\pi(1), x_{n}=\pi\left(\alpha^{n}\right), y_{n}=$ $\pi\left(\alpha^{n-1} \gamma\right), n \in \mathbf{Z}_{\geq 1}$. We conjecture that also for this case $S_{q}^{2}(\mu, \mu) \cong S U_{q}(2)^{c o C}$.

\section{Conclusions}

In this paper we have shown that certain embeddable quantum homogeneous 
embeddable spaces include the general quantum two-sphere $S_{q}^{2}(\mu, \nu)$ and the quantum plane $\mathbf{C}_{q}^{2}$. The interpretation of quantum embeddable spaces presented in this paper seems specially interseting from the point of view of quantum group gauge theory, the suitable generalisation of which we have also proposed. We think that it would be intersting and indeed desirable to develop further this generalisation of quantum group gauge theory, and in particular, to construct connections on the quantum spaces described in this paper. For example this would allow for extending the construction of the Dirac $q$-monopole of $[\mathrm{BM}]$ to general quantum spheres.

Acknowledgement. I would like to thank Shahn Majid for suggesting to me the proofs of Propositions 2.4 and 2.5(2).

\section{REFERENCES}

[BM] T. Brzeziński and S. Majid, Quantum Group Gauge Theory on Quantum Spaces, Commun. Math. Phys. 157 (1993), 591; ibidem. 167 (1995), 235. (Erratum)

[CP] V. Chari and A. Pressley, A Guide to Quantum Groups, Cambridge University Press, 1994.

[C] A. Connes, A lecture given at the Conference on Non-commutative Geometry and Its Applications, Castle Třešt. Czech Republic, May 1995.

[M] Y.I. Manin, Quantum Groups and Non-commutative Geometry, Montreal Notes, 1989.

[NM] M. Nuomi and K. Mimachi, Quantum 2-spheres and Big q-Jacobi Polynomials, Commun. Math. Phys. 128 (1990), 521.

[P1] P. Podleś, Quantum Spheres, Lett. Math. Phys. 14 (1987), 193.

[P2] - Symmetries of Quantum Spaces. Subgroups and Quotient Spaces of Quantum $S U(2)$ and $S O(3)$ Groups, Commun. Math. Phys. 170 (1995), 1.

[S] H.-J. Schneider, Principal homogeneous spaces for arbitrary Hopf algebras, Israel J. Math 72 (1990), 167; Representation theory of Hopf-Galois extensions, ibidem. 72 (1990), 196.

[Sw] M.E. Sweedler, Hopf algebras, Benjamin, 1969.

Institute of Mathematics, University of Łódź, ul. Banacha 22, 90-238 Łódź, POLAND

Current address: University of Cambridge, DAMTP, Cambridge CB3 9EW, UK. (after 1st October 1995)

E-mail address: tbrzez@ ulb.ac.be or t.brzezinski@ damtp.cam.ac.uk 